\title{
Métodos de Repartição dos Custos Indirectos utilizados pelas PMEs industriais portuguesas
}

\author{
Product Costing in Small and Medium Sized Portuguese Manufacturing Companies
}

\author{
Maria João Machado \\ Doutora em Contabilidade no ISCTE-Instituto Universitário de Lisboa \\ Professora Auxiliar do Departamento de Contabilidade do ISCTE-Instituto Universitário de Lisboa \\ Endereço: Avenida das Forças Armadas, 1649-026 Lisboa, Portugal \\ E-mail: maria.joao.machado@iscte.pt \\ Telefone: (011) (351) 217-903950
}

Artigo recebido em janeiro de 2009. Passou por uma avaliação double blind review em junho de 2009. Aceito em setembro de 2009 pela Editora Científica Sandra Rolim Ensslin. Artigo apresentado no XII Congresso de Contabilidade e Auditoria, 2008, Aveiro, 2008. 


\title{
Resumo
}

O presente trabalho tem por objectivo caracterizar a forma como são tratados os custos indirectos nas empresas industriais portuguesas, classificadas pelo Instituto de Apoio às Pequenas e Médias Empresas e ao Investimento como PME excelência, para efeitos de valorização dos produtos. Os pressupostos ontológicos e epistemológicos subjacentes a este estudo são compatíveis com o paradigma de investigação positivista. Foram realizadas entrevistas a responsáveis pela Contabilidade de Gestão de 58 empresas localizadas em 11 Distritos portugueses. A evidência recolhida permite concluir que todas as empresas analisadas utilizam bases de repartição dos custos indirectos que são influenciadas pelo volume de produção. Todas as bases de imputação encontradas são influenciadas pela quantidade produzida de cada produto, o que provoca a sobrevalorização dos produtos com maior volume de produção. Se o custo dos produtos for utilizado para efeitos de tomada de decisão, nomeadamente para determinação do preço de venda ou análise de rendibilidade de cada produto, pode ter implicações estratégicas importantes na empresa, na medida em que os gestores podem conduzir erradamente os recursos para os produtos com menor volume.

Palavras-chave: Custos Indirectos, PME, Portugal

\begin{abstract}
This study aims to characterize the way in which some indirect costs in the Portuguese industrial firms, ordered by the Institute of Support to Small and Medium Enterprises and Investment as SMEs excellence, for recovery of products. The ontological and epistemological assumptions underlying this study are consistent with the positivist research paradigm. Interviews were conducted by the responsible Management Accounting from 58 companies located in 11 districts Portuguese. The evidence collected to suggest that all companies analyzed using bases for allocating indirect costs that are influenced by the volume of production. All bases of imputation found are influenced by much of each product, which causes the overvaluation of the products with higher production volume. If the cost of products is used for decision-making, especially for determining the selling price or profitability analysis of each product, can have important strategic implications in the workplace, to the extent that managers can drive the wrong resources to products with less volume.
\end{abstract}

Keywords: Indirect costs, SMEs, Portugal

\section{Introdução}

O presente trabalho tem por objectivo contribuir para o conhecimento sobre a forma como as Pequenas e Médias Empresas (PMEs) industriais portuguesas repartem os custos indirectos, para efeitos de valorização dos produtos. Como objectivos mais específicos, podem-se identificar os seguintes: (i) Caracterizar os métodos utilizados nas empresas industriais classificadas como PME excelência, de forma consistente, neste século XXI. Essa classificação é atribuída pelo Instituto de Apoio às Pequenas e Médias Empresas e ao Investimento (IAPMEI); (ii) Identificar as bases de repartição dos custos indirectos mais utilizadas, analisando se são influenciadas pelo volume de produção.

Restringiu-se o universo a estudar às PMEs, devido ao seu elevado peso no tecido empresarial nacional. Um estudo realizado pelo IAPMEI (2002) refere que 99,5\% das empresas nacionais são pequenas e médias, o que confere relevância ao estudo desse tipo de empresas para caracterizar a situação do País. 
No entanto, o elevado número de PMEs em Portugal, 1.140.735 empresas em 1999 (IAPMEI, 2002), exige uma maior restrição do universo a analisar. Dessa forma, delimitou se este estudo às empresas classificadas pelo IAPMEI como excelência-industria, por duas razões: (i) A delimitação às PMEs industriais justifica-se por estas pertencerem ao sector de actividade que, em termos históricos, tem mais tradição na utilização de métodos de repartição dos custos indirectos, para efeitos de valorização dos produtos; (ii) A utilização de empresas classificadas como PME excelência justifica-se pelo facto de constituir uma selecção de empresas já realizada com objectivos convergentes a este trabalho, uma vez que a referida classificação pretende "...distinguir as pequenas e médias empresas que mais se destacam... pelos seus desempenhos económico-financeiro e de gestão..." (IAPMEI, 2002, p. 17).

Relativamente ao período da análise, optou-se pela delimitação do universo a estudar as empresas que obtiveram essa classificação de forma consistente em 2000 e 2001. As justificações para esta opção são duas: (i) O objectivo de tratar a informação mais recente levou à opção pelos dois últimos anos de atribuição da classificação; (ii)A selecção das empresas classificadas simultaneamente nesses dois anos tem como objectivo identificar aquelas que corresponderam de forma consistente aos requisitos do prémio. Com esse procedimento, excluem-se da análise aquelas empresas que podem ter conseguido pontualmente atingir os requisitos recorrendo a manobras de Engenharia Financeira.

Os pressupostos ontológicos e epistemológicos subjacentes a este trabalho são compatíveis com o paradigma de investigação positivista.

O universo em análise é constituído pelas 163 empresas classificadas de forma consistente, no século XXI, como PMEs excelência - indústria. A recolha de dados foi feita pela realização de entrevistas aos responsáveis pela Contabilidade de Gestão por se considerar que estes têm o domínio da informação sobre a qual se pretende recolher evidência. Foram realizadas 58 entrevistas em empresas localizadas em 11 Distritos de Portugal, o que gerou uma taxa de resposta de 36\%. Foi realizado o tratamento da não resposta, que permitiu concluir não existirem diferenças estatisticamente significativas entre as empresas respondentes e as não respondentes.

\section{Revisão de Literatura}

A informação necessária à gestão de qualquer organização exige a valorização de diversos objectos de custo, os quais são definidos por Franco et al. (2005, p. 215) como "entidades (produtos, serviços, actividades, departamentos, clientes, ...) para as quais se considera necessário conhecer o respectivo custo".

Para a adequada valorização dos objectos de custo é necessário atribuir- 
lhes, da forma mais rigorosa possível, todos os recursos por eles consumidos (FRANCO et al., 2005), os quais podem ser de dois tipos (HORNGREN et al., 1997, p. 27):

- Custos directos - são aqueles que podem ser relacionados directamente com um objecto de custo em particular de uma forma economicamente viável.

- Custos indirectos - são aqueles que não podem ser relacionados directamente com um objecto de custo em particular de uma forma economicamente viável. Estes têm de ser repartidos (allocated) (HORNGREN et al., 1997, p. 27) pelos diversos objectos de custo, através de um método de repartição definido pela empresa.

A classificação dos custos como directos ou indirectos depende do objecto de custo que se pretende valorizar (FRANCO et al., 2005), o que exige a sua prévia definição. O presente trabalho centra-se na repartição do último tipo de custos, diante de um objecto de custo em particular, os produtos fabricados pelas empresas industriais. A relevância dessa problemática justifica-se pela necessidade de a Contabilidade fornecer informação útil à gestão (FERREIRA, 2007), o que torna a adequada repartição dos custos indirectos essencial para a tomada de decisão, quer em termos de pricing, quer para a análise da rendibilidade de cada produto.

Horngren (2004) considera que um dos dilemas da Contabilidade de Gestão nas empresas industriais do século XX foi a problemática relação de causa-efeito entre os custos indirectos e os produtos, serviços ou clientes. Chauvey (1997) concorda com essa posição ao considerar que um dos problemas centrais da análise de custos é saber como repartir os custos indirectos. Mevellec (2001) traduz a mesma preocupação ao referir que o potencial de um sistema de apuramento de custos está intimamente relacionado com a sua capacidade para modelar de forma fidedigna as relações de causalidade entre o consumo de recursos e a produção.

A preocupação de encontrar as bases de repartição mais adequadas para relacionar os custos indirectos com os objectos de custo levou à criação de diversos métodos, desde a utilização de simples coeficientes de imputação, repartição por departamentos e repartição por actividades (HORNGREN, 2004). Essa evolução levou à passagem da utilização de uma única base para múltiplas bases de repartição, numa tentativa sistemática de melhorar a relação entre as bases escolhidas e os factores que provocam a existência do custo.

\section{a) - A repartição dos custos indirectos por meio de coeficientes de imputa- ção}

A forma mais simples de repartir por produtos os custos indirectos é utilizando uma única base de repartição (FRANCO et al., 2005). Nesse método, a 
repartição dos custos indirectos é feita por meio de um coeficiente de imputação. Trata-se de um quociente em que, no numerador, figura o total de custos indirectos que se pretende repartir e, no denominador, a base de repartição escolhida pela empresa. Esta base deve estar fortemente relacionada com o factor que origina os custos indirectos (PEREIRA e FRANCO, 1991).

As bases de repartição mais utilizadas são os próprios consumos directos, nomeadamente o valor da matéria directa, a mão-de-obra directa em valor ou em quantidade (hora-homem) e o número de horas de trabalho das máquinas (horamáquina) (COOPER, 1987b).

A dificuldade de encontrar uma única base de repartição que traduza uma relação de causa efeito com os factores que provocam a existência dos custos levou ao desenvolvimento da repartição de base múltipla. Nesse método, os custos indirectos são divididos em diversos grupos, com características homogéneas, de forma a simplificar a escolha de uma base de repartição mais adequada ao comportamento de cada grupo. A quota de custos indirectos de cada produto tem uma parcela de cada coeficiente, ou seja, de cada grupo definido, o que faz com que a repartição de base múltipla permita um cálculo mais rigoroso do custo de cada produto do que o resultante da utilização de uma única base (FRANCO et al., 2005).

Embora seja melhor, a repartição de base múltipla é ainda alvo de muitas criticas, nomeadamente Cooper (1987b e 1988); Johnson e Kaplan (1991); Kaplan (1998); e Dearman e Shields (2001).

Embora Cooper (1987a e 1987b) reconheça que a utilização de outras bases de repartição como a hora-máquina ou o valor das matérias, para além da mão-deobra directa, tenha melhorado a repartição dos custos indirectos, elas continuam a não ser adequadas, pois todas são influenciadas pela quantidade produzida de cada produto. Cooper (1988) demonstra, por meio de exemplos, que, quando existem grandes diferenças de volume de produção entre os produtos, os que se produzem em maior quantidade são os que consomem mais bases de repartição relacionadas com esse factor, sejam elas a matéria directa, a mão-de-obra directa ou a hora-máquina. Sendo estes os critérios para se repartirem os custos indirectos, são os produtos que mais consomem dessas bases os que ficam com maior parcela desses custos. Na maioria dos processos de fabrico, existem custos que não são originados pelo volume de produção, como os custos de inspecção ou de preparação das máquinas, os quais têm de ser repartidos pelos produtos por outro tipo de bases não influenciadas pela quantidade produzida. Continuar a utilizar as bases tradicionais para repartir esses custos provoca uma distorção no custo dos produtos (COOPER, 1988). Por exemplo, se muitos dos custos indirectos se relacionarem com o número de ordens de produção, o custo dos produtos com poucas ordens, mas com muitas quantidades em cada uma delas, fica sobreva- 
lorizado. Em contrapartida, o custo dos produtos fabricados por meio de muitas ordens de produção, mas com pouca quantidade em cada uma delas, fica subvalorizado. Dearman e Shields (2001) generalizam essa distorção para outros factores. Estes autores referem que muitas empresas repartem os custos indirectos pelos produtos utilizando bases relacionadas com o volume, como a hora-homem e a hora-máquina, as quais provocam uma distorção no custo dos produtos quando estes têm diferenças significativas em termos de consumo de recursos, diferenças essas não relacionadas com a quantidade produzida.

Cooper (1987b) defende que esses métodos não podem continuar a ser utilizados, pois os custos não relacionados com o volume já chegam a atingir os $25 \%$ do total do custo industrial. Essa distorção no custo dos produtos é importante para a gestão das empresas, na medida em que faz com que os gestores tenham dificuldade em definir qual a melhor afectação dos recursos disponíveis, diante dos múltiplos produtos existentes. Essa opinião é idêntica à de Kaplan (1998) e Johnson e Kaplan (1991). Estes autores consideram que a repartição dos custos indirectos exige o reconhecimento de que muitos deles não são provocados pela quantidade de produto, mas, sim, por outros factores como o registo de uma nova encomenda, a preparação do processo de fabrico para cada novo lote produtivo, a recepção e inspecção de matérias, entre outros.

No entanto, os estudos empíricos revistos reportam a utilização do método mais simples de repartição dos custos indirectos. Encontraram-se diversos artigos que descrevem a realidade de sete países (Reino Unido, Irlanda, Portugal, Finlândia, Malásia, Estónia e Índia), os quais são apresentados no Quadro1.

\section{Quadro1 - A problemática das bases de repartição relacionadas com o volume}

\begin{tabular}{|l|l|l|c|c|c|} 
Artigo & País & $\begin{array}{l}\text { Método de } \\
\text { recolha de } \\
\text { dados }\end{array}$ & $\begin{array}{c}\text { Número de } \\
\text { respondentes }\end{array}$ & $\begin{array}{c}\text { Base de } \\
\text { repartição } \\
\text { única }\end{array}$ & $\begin{array}{c}\text { Base de } \\
\text { repartição mais } \\
\text { utilizada }\end{array}$ \\
\hline \hline Drury e Tayles (1994) & $\begin{array}{l}\text { Reino } \\
\text { Unido }\end{array}$ & Inquéritos & 303 & $26 \%$ & MOD \\
\hline Clarke (1997) & Irlanda & Inquéritos & 204 & --- & MOD \\
\hline $\begin{array}{l}\text { Lukka e Granlund } \\
(1996)\end{array}$ & Finlândia & Inquéritos & 135 & $5 \%$ & --- \\
\hline Chun et al. (1996) & Malásia & Inquéritos & 92 & $32 \%$ & MOD \\
\hline $\begin{array}{l}\text { Joshi (2001) } \\
\text { Índia }\end{array}$ & Inquéritos & 60 & $35 \%$ & MOD \\
\hline Raldma e Lääts (2002) & Estónia & Inquéritos & 62 & --- & MOD \\
\hline Rodrigues et al. (2001) & Portugal & Inquéritos & 127 & --- & MOD \\
\hline
\end{tabular}

Fonte: Dados da pesquisa.

Drury e Tayles (1994) realizaram inquéritos sobre as práticas de valoriza- 
ção dos produtos em 303 divisões de 260 grandes empresas industriais do Reino Unido. Os resultados mostraram que $26 \%$ dos respondentes utilizam uma única base de imputação para repartir os custos indirectos. Independente de terem ou não processos de fabrico automatizados, a base de imputação mais utilizada é a Mão-de-Obra Directa (MOD), em 73\% das empresas automatizadas, e 68\% das não automatizadas. Os autores criticam a utilização desta base de imputação, principalmente quando se utilizam processos de fabrico automatizados.

Clarke (1997) utilizou inquéritos postais aos responsáveis pela Contabilidade de Gestão para recolher evidência sobre as práticas de apuramento do custo dos produtos em 204 grandes empresas industriais irlandesas. O autor confirma os resultados de Drury e Tayles (1994 e 1995) ao concluir que a base de imputação mais utilizada é a Mão-de-Obra Directa (MOD), embora seja a componente do custo industrial com menor peso. O estudo reporta que a componente fundamental do custo dos produtos são as matérias consumidas, mas estas só são utilizadas para repartir os custos indirectos em $7 \%$ dos casos.

Lukka e Granlund (1996) realizaram inquéritos postais aos responsáveis pela Contabilidade de Gestão de 135 empresas industriais finlandesas de média e grande dimensão e concluem que a maior parte das empresas reparte os custos indirectos industriais utilizando bases de imputação relacionadas com o volume. A maioria das empresas utiliza diversas bases de imputação em simultâneo, e apenas $5 \%$ utilizam uma única base. A repartição dos custos indirectos não industriais é realizada por meio de diversos critérios, sendo o mais usual o peso dos custos industriais, seguindo-se o valor das vendas.

Chun et. al. (1996) realizaram inquéritos às práticas de Contabilidade de Gestão de 92 empresas industriais na Península da Malásia. Recorrendo a um directório nacional, foram seleccionadas indústrias específicas de sectores mais inovadores e competitivos nos quais é exigida mais tecnologia de produção. Os resultados mostram que 32\% dos respondentes utilizam uma única base de repartição dos custos indirectos, o que é compatível com o estudo de Drury e Tayles (1994). A mão-de-obra directa é a base de imputação mais utilizada (51\%), seguindo-se a quantidade produzida (21\%) e o número de horas de trabalho das máquinas (19\%), sendo o valor das matérias consumidas a base de imputação menos utilizada (9\%). Esses autores analisam ainda a natureza real ou standard das bases, concluindo que os standard são muito utilizados (36\%), nomeadamente a hora-homem standard que representa $17 \%$, o valor standard da mão-de-obra directa que representa $10 \%$ e a hora-máquina standard que representa $9 \%$.

Joshi (2001) realizou inquéritos às práticas de Contabilidade de Gestão de 60 grandes e médias empresas industriais na Índia. Relativamente ao sistema de valorização dos produtos, o autor refere que $35 \%$ das empresas utilizam uma única base de repartição dos custos indirectos, sendo a mão-de-obra directa a 
mais utilizada.

Haldma e Lääts (2002) realizaram inquéritos a responsáveis de 62 grandes empresas industriais da Estónia sendo que estes também concluem que a mão-deobra directa é a base mais utilizada para repartir os custos indirectos.

Rodrigues et al. (2001) analisaram as práticas de Contabilidade de Gestão em PMEs Portuguesas, por meio de inquéritos a 127 empresas classificadas como PME excelência. Relativamente às empresas industriais, 64\% imputam aos produtos os custos indirectos por meio de coeficientes de imputação, não referindo, no entanto, se utilizam base única ou múltipla. O estudo refere que a mão-de-obra directa é a base de imputação mais utilizada.

A revisão dos artigos anteriores permite concluir que a maior parte das empresas utiliza bases de repartição dos custos indirectos influenciadas pela quantidade produzida, sendo a mão-de-obra directa a mais utilizada.

\section{b) - A análise por secções}

O sistema de Contabilidade de Gestão francês, relativo ao tratamento dos custos indirectos, tem-se mantido praticamente inalterado desde a publicação em Paris, em 1937, do "Méthode des Sections Homogènes" (LEBAS, 1994, p. 473) pela CEGOS - Commission Générale d' Organisation Scientifique, criada por uma federação de empresas industriais. O principal objectivo foi fornecer aos seus associados um plano de Contabilidade de Gestão standard que lhes permitisse competir melhor entre si. Como não existia uma organização profissional que representasse os contabilistas de gestão em França, foram as associações industriais que tiveram um papel activo no desenvolvimento das técnicas de Contabilidade de Gestão utilizadas nesse país.

O governo francês foi muito intervencionista nas práticas de Contabilidade de Gestão, tendo um importante papel na divulgação deste método. O trabalho inicial sobre a normalização da contabilidade em França foi realizado, em 1947, por uma comissão semigovernamental, a Commission interministérielle de normalisation des comptabilités (LEBAS, 1994). Esse trabalho inicial foi revisto em 1957 e em 1982 por uma comissão que funcionava nas dependências do Ministério das Finanças e da Economia, o denominado Conseil National de la Comptabilité (LEBAS, 1994).

O actual Plano de Contabilidade Francês em vigor é de 1982, denominado Plan Comptable Général (LEBAS, 1994), sendo o seu objectivo fundamental a normalização da contabilidade externa. No entanto, com o objectivo de melhorar o desempenho empresarial, esse Plano Contabilístico apresenta um capítulo sobre contabilidade interna meramente indicativo, por meio do qual se aconselham as 
empresas a organizar um sistema de contabilidade interna baseado no método das secções homogéneas desenvolvido pela CEGOS. O Plano Contabilístico Francês descreve o, relativo ao apuramento do custo dos produtos, da seguinte forma:

- Este método baseia-se na divisão de toda a estrutura organizacional em unidades básicas, designadas por centros de análise. Lebas (1994) refere que a tradição francesa é chamar-lhes secções ou centros de responsabilidade. Rocha e Rubio (1999, p. 305) definem secção como "um agrupamento real ou fictício de encargos, estabelecido de tal modo que o total dos encargos que a constituam pode ser referido a uma unidade comum que permita o seu controlo e a sua ulterior afectação ao produto".

- Cada secção deve ter uma e uma só unidade de medida (ROBERTS, 1995), à qual se chama unité d'oeuvre (LEBAS, 1994). É recomendado que a unidade de obra seja expressa em unidades físicas como a hora-homem ou a hora-máquina. Só é permitida a utilização de critérios de imputação baseados na percentagem de outros custos quando não exista outra alternativa, ou seja, quando as características da secção não permitem que lhe seja atribuída uma unidade física para medida da sua actividade.

- Segundo Lebas (1994), o método não obriga à classificação clássica das secções por áreas funcionais da empresa, nomeadamente como industriais, administrativas e de distribuição.

- As secções consomem recursos, com excepção dos custos directos dos produtos (ROCHA e RUBIO, 1999).

- Os recursos utilizados por cada secção são consumidos com vista à obtenção da sua unidade de obra. Os utilizadores desse output devem então receber o custo inerente a essa utilização de acordo com a quantidade de unidade de obra que consumiram (LEBAS, 1994).

- As secções são classificadas em termos de quem são os beneficiários da sua actividade. Se são os produtos, denominam-se secções principais. Se são outras secções, denominam-se secções auxiliares. Os custos destas últimas são distribuídos pelas secções principais antes do apuramento do custo dos produtos (PEREIRA e FRANCO, 1991).

Comparando os dois métodos anteriores, pode-se concluir que o método das secções homogéneas permite apurar de forma mais rigorosa o custo de cada produto, ao melhorar a relação de causa-efeito entre os produtos e os seus custos indirectos, por meio da unidade de obra de cada secção efectivamente utilizada por cada um deles. Para além dessa vantagem, esse método permite avaliar e controlar os custos de cada secção, permitindo ao gestor analisar a sua eficiência e eficácia e ponderar a sua existência na empresa. 


\section{c) - A análise por actividades}

A análise por actividades surgiu com a construção de um novo sistema de custeio, iniciada em meados da década de 1980, que ficou conhecido como Activity Based Costing. Para o seu desenvolvimento, contribuíram significativamente duas organizações: a Harvard Business School, por intermédio de dois dos seus investigadores, Robert Kaplan e Robin Cooper, e uma organização de investigação e desenvolvimento, denominada Computer-Aided Manufacturing, International (CAM-I), financiada por empresas industriais e agências governamentais (Jones e Dugdale, 2002).

A valorização dos produtos no custeio, baseado nas actividades, é caracterizada pelos seguintes aspectos:

- A empresa é estruturada em actividades. São diversas as definições de actividades, desde uma abordagem mais teórica que considera como actividade todo trabalho que consome recursos e acrescenta valor a um negócio (JOHNSON, 1988), até uma definição mais prática de que uma actividade é composta por um conjunto de tarefas elementares relacionadas entre si (FRANCO et al., 2005).

- Tal como no método das secções homogéneas, os custos da empresa são divididos em duas categorias. Os custos directos dos produtos, os quais são relacionados directamente com os produtos apropriados (COOPER, 1990). Os custos indirectos dos produtos, os quais são relacionados com as actividades (COOPER e KAPLAN, 1988).

- Cada actividade tem de ter uma unidade de medida, à qual Cooper (1990) chama condutor de custo (cost driver).

- Os utilizadores das actividades são os objectos de custo, os quais recebem o custo inerente a essa utilização de acordo com a quantidade de condutor de custo que utilizam (COOPER e KAPLAN, 1988).

Vários são os autores que atribuem ao custeio baseado nas actividades uma supremacia evidente relativamente à determinação do custo de cada produto. Essa resulta da forma como são tratados os custos que não estão relacionados com o volume de produção, mas, sim, com outros factores como o número de ordens de produção ou o número de produtos (COOPER, 1988). Os outros métodos de valorização dos produtos repartem os custos indirectos por meio de bases de repartição influenciadas pelo volume de produção, o que pode não reflectir o verdadeiro consumo de recursos organizacionais requeridos pelos produtos (SWENSON, 1995). Esta característica dos outros métodos provoca a sobrevalorização dos produtos fabricados em grandes quantidades e a subvalorização dos produtos 
fabricados em pequenas quantidades (JOHNSON, 1988). A informação por eles fornecida apenas pode ser utilizada para efeitos de valorização de inventários na Contabilidade Financeira, mas não para efeitos de tomada de decisão. Caso contrário, a sua utilização tem implicações estratégicas importantes, pois leva os gestores a conduzirem erradamente os recursos para os produtos com menor volume de produção (JOHNSON, 1988).

Kennedy e Affleck-Graves (2001) analisam, por meio de uma amostra de 47 empresas do Reino Unido, que à introdução do custeio baseado nas actividades está associada uma avaliação mais favorável da empresa, quer utilizando indicadores de Contabilidade de Gestão, quer por indicadores do mercado financeiro. Estes autores defendem que o custeio baseado nas actividades permite uma melhor atribuição dos custos aos produtos por meio de uma melhor repartição dos custos indirectos por via da identificação e compreensão dos condutores de custo das actividades.

Apesar dos benefícios teóricos resultantes da sua utilização, Gosselin (1997) identifica o paradoxo da abordagem das actividades, o qual está relacionado com a seguinte interrogação: Se este método proporciona tantos benefícios à gestão das empresas, por que é que os estudos empíricos não apresentam taxas de utilização mais elevadas? São diversos os estudos empíricos que reportam taxas de utilização do custeio baseado nas actividades em diversos países. Na maior parte dos países, encontram-se taxas de utilização iguais ou inferiores a $20 \%$, nomeadamente no Reino Unido (DRURY e TAYLES, 1994; INNES e MITCHELL, 1995; INNES et al., 2000); na Nova Zelândia (COTTON e JACKMAN, 2002) no Japão (HOPPER et al., 1999); na Índia (JOSHI, 2001); na Irlanda (CLARKE e O' DEA, 1994; CLARKE et al., 1999); em Espanha (BLAKE et al., 2000); em Singapura (GHOSH e CHAN, 1997); na Malásia (CHUN et al., 1996); em Portugal (RODRIGUES et al., 2001); na Estónia (HALDMA e LÄÄTS, 2002); e na Finlândia (LUKKA e GRANLUND, 1996).

A resposta a este paradoxo pode estar nas dificuldades associadas à sua implementação:

- A dificuldade de identificar as actividades e de seleccionar os condutores de custo mais adequados a cada uma delas. Mevellec (2001) considera esta a principal justificação para a baixa taxa de utilização nas empresas francesas. Este problema também é identificado por Chauvey (1997), quando se refere à necessidade de se definir o nível de detalhe adequado para a identificação das actividades. Embora se saiba que uma actividade é composta por um conjunto de tarefas elementares relacionadas entre si, não é fácil, na prática empresarial, determinar o número de tarefas que devem compor uma actividade.

- A dificuldade de apurar o custo de cada actividade. Chauvey (1997) 
considera que o custeio baseado nas actividades é um método sedutor em termos teóricos, pois assenta na ideia de que a relação entre os custos indirectos e os produtos é muito mais adequada do que nos outros métodos, uma vez que as actividades permitem identificar melhor a relação de causa-efeito entre os recursos consumidos e os objectos de custo. No entanto, as dificuldades práticas da sua implementação, nomeadamente a repartição dos custos comuns a diversas actividades, conduzem a aproximações e distorções que podem eliminar as suas vantagens diante dos outros métodos, em termos de valorização dos produtos.

- A resistência à mudança por parte dos trabalhadores. Major e Hooper (2005) analisaram, por meio de um estudo de caso numa empresa portuguesa, que a implementação da abordagem por actividades é muito condicionada por factores comportamentais, uma vez que os trabalhadores desempenham um papel fundamental no sucesso do sistema. Se os trabalhadores considerarem o novo sistema como uma ameaça à sua autonomia ou à segurança do posto de trabalho, podem sabotar a sua implementação.

\section{Questão de Estudo}

A forma como se adquire conhecimento acerca de um fenómeno é condicionada pelo modo como se vê a realidade, o que significa que a posição epistemológica do investigador tem de ser coerente com a sua posição ontológica. Da mesma forma, a metodologia de investigação escolhida tem de ser coerente com a posição epistemológica do investigador. Com base nesses três factores, vários autores classificam a investigação em Contabilidade pela identificação de grandes paradigmas. Bhimani (2002, p. 101) define paradigma como “... a rota teórica de uma perspectiva de investigação...”.

Identificaram-se, na literatura revista, três grandes paradigmas de investigação em Contabilidade. O funcionalismo, também conhecido por positivismo, é defendido por diversos autores, nomeadamente Watts e Zimmerman (1990), Chambers (1993) e Zimmerman (2001). O interpretativismo ou investigação interpretativa é defendido por Scapens (1990), Humphrey e Scapens (1996), Covaleski e Dirsmith (1988) e Burns e Scapens (2000), entre outros. A investigação crítica é defendida por autores como Miller e O’ Leary (1987), Laughlin (1987) e Hopper e Armstrong (1991).

Uma análise crítica sobre as vantagens e desvantagens da utilização dos diferentes paradigmas de investigação em Contabilidade permitiu concluir que não é possível identificar qual o mais adequado, pois todos eles apresentam desvantagens. Existem actualmente muitas posições consensuais relativas à utilização de diferentes paradigmas, nomeadamente Covaleski e Dirsmith (1996), Bhimani (2002), Chua (1986), e Lukka e Granlund (2002). Optou-se, neste estudo, pela 
utilização do paradigma de investigação positivista por se considerar que o tipo de evidência que se pretende obter é compatível com uma concepção objectiva da realidade, considerando-a como algo externo ao investigador, características estas associadas a este paradigma de investigação (CHUA, 1986).

Com base nesse paradigma de investigação, formulou-se a seguinte questão de estudo: As PMEs industriais portuguesas utilizam métodos de repartição dos custos indirectos influenciados pelo volume de produção?

São dois os objectivos a atingir com a formulação desta questão. $O$ primeiro é conhecer a forma como as empresas repartem os custos indirectos e comparar com os métodos utilizados noutros países. O segundo é identificar as bases de repartição mais utilizadas e analisar se são influenciadas pelo volume de produção.

\section{Método de Pesquisa}

Relativamente ao método de recolha de dados a utilizar neste trabalho, a questão de pesquisa anteriormente definida limitou as opções para duas: inquéritos ou entrevistas. Tornou-se então necessário definir qual das duas seria a mais adequada. Abernethy et al. (1999) defendem que não existe um método de pesquisa ideal, todos eles apresentam vantagens e desvantagens. No entanto, esses autores defendem que o mais importante é encontrar o método mais adequado para recolher evidência sobre as questões de pesquisa definidas pelo investigador. Criticam o facto de, muitas vezes, o método ser considerado pelos investigadores como um fim em si mesmo e não como um meio para obter a melhor recolha de evidência para as questões em análise.

Como principais vantagens da utilização de entrevistas para recolher evidência sobre as questões formuladas neste trabalho, podemos identificar as seguintes:

- As matérias sobre as quais se pretende recolher evidência não se encontram normalizadas em Portugal, o que pode gerar diferentes denominações para a mesma realidade nas diferentes empresas. A utilização de entrevistas permite que os conceitos sejam explicados aos entrevistados o que gera uma maior fiabilidade das respostas (BELL, 2002). As entrevistas semiestruturadas são particularmente úteis para recolher informação sobre a qual não há a certeza de todos os intervenientes compreenderem os conceitos que o investigador tenta avaliar (ABERNETHY et al., 1999).

- Dado que as práticas de Contabilidade interna das PMEs portuguesas são uma realidade pouco conhecida, a utilização de entrevistas permite obter informação adicional, não prevista inicialmente pelo investigador, que pode revelar-se útil para o próprio trabalho ou para outros futuros (BELL, 2002). 
Os inquéritos são um método adequado para recolher informação sobre conceitos estandardizados, aceites e partilhados por todos os intervenientes, $\mathrm{o}$ que não se aplica às informações necessárias neste trabalho (Abernethy et al., 1999). O facto de os inquiridos não terem oportunidade de clarificar dificuldades interpretativas pode influenciar os resultados da própria investigação.

No entanto, a utilização de entrevistas para recolher evidência sobre as questões deste trabalho também apresenta desvantagens diante da realização de inquéritos:

- O facto de a informação ser recolhida a partir de uma única fonte. Essa desvantagem é superada pela realização de inquéritos quando são inquiridas diversas fontes dentro da mesma empresa. No entanto, Abernethy et al. (1999) consideram que esta desvantagem pode ser superada quando o investigador tem a certeza de que o entrevistado tem o domínio da informação sobre a qual se pretende recolher evidência.

- As entrevistas consomem mais tempo do que os inquéritos (BELL, 2002), principalmente se forem realizadas em amplas áreas geográficas.

- As entrevistas potenciam a possibilidade do entrevistador induzir a própria resposta do entrevistado (BELL, 2002), o que as torna um método mais subjectivo do que a realização de inquéritos. Abernethy et al. (1999) consideram que este factor não produz mais enviesamento na pesquisa do que o resultante da subjectividade de interpretação dos questionários.

Diante do tipo de questões de investigação colocadas neste estudo, optou-se pela realização de entrevistas que permitissem recolher evidência, a mais variada possível, sobre as questões levantadas. Dessa forma, pretendeu-se recolher informação mais rica, na medida em que da própria entrevista poderiam surgir variáveis não previstas inicialmente, assim como evidência mais fidedigna na medida em que os conceitos básicos associados à Contabilidade de Gestão podem não ser utilizados da mesma forma por todas as empresas portuguesas. Optou-se pela realização de entrevistas semiestruturadas nas quais não se utiliza um questionário de base à entrevista, mas, sim, um conjunto de tópicos sobre os quais esta tem de incidir (BELL, 2002).

As entrevistas foram dirigidas apenas ao responsável pela contabilidade interna por se considerar que este tem o domínio da informação sobre a qual se pretendia recolher evidência.

\section{Recolha de Dados}

Este trabalho pretende analisar o universo das PMEs industriais portuguesas classificadas como excelência pelo IAPMEI em 2000/2001. Apenas 163 
empresas o conseguiram obter de forma consistente nos dois últimos anos da sua atribuição, constituindo assim o universo deste estudo.

Após três fases de contacto, conseguiu-se a marcação de reuniões em 58 empresas. As entrevistas foram realizadas com o responsável pela contabilidade interna entre julho de 2005 e julho de 2006. A distribuição geográfica das empresas que aceitaram participar é apresentada no Quadro2.

Quadro2 - Cobertura geográfica das entrevistas realizadas

\begin{tabular}{||l|c|c|c||} 
Distrito & $\begin{array}{c}\mathrm{N}^{\circ} \text { empresas } \\
\text { no universo }\end{array}$ & $\begin{array}{c}\mathrm{N}^{\circ} \text { entrevistas } \\
\text { realizadas }\end{array}$ & $\%$ do universo \\
\hline Braga & 21 & 3 & $14 \%$ \\
\hline Vila Real & 1 & 0 & $0 \%$ \\
\hline Porto & 28 & 4 & $14 \%$ \\
\hline Aveiro & 46 & 6 & $13 \%$ \\
\hline Viseu & 7 & 7 & $100 \%$ \\
\hline Coimbra & 10 & 3 & $30 \%$ \\
\hline Castelo Branco & 1 & 0 & $0 \%$ \\
\hline Leiria & 23 & 14 & $61 \%$ \\
\hline Santarém & 4 & 2 & $50 \%$ \\
\hline Lisboa & 13 & 11 & $85 \%$ \\
\hline Setúbal & 6 & 6 & $100 \%$ \\
\hline Évora & 1 & 1 & $100 \%$ \\
\hline Faro & 1 & 1 & $100 \%$ \\
\hline Ponta Delgada & 1 & 0 & $0 \%$ \\
\hline
\end{tabular}

Fonte: Dados da pesquisa.

Foram analisadas empresas de 11 dos 14 distritos do universo inicial, pelo que se considera ter obtido uma boa cobertura geográfica do universo em análise.

As 58 empresas que aceitaram participar correspondem a uma taxa de resposta de $36 \%$, o que é comparável à de outros trabalhos analisados na revisão de literatura, nomeadamente Drury e Tayles (1994) que apresentam uma taxa de resposta de 35\%, Haldma e Lääts (2002) têm uma taxa de resposta de 34\%, Innes e Mitchell (1995), Innes et al. (2000), Joshi (2001), com taxas de resposta de apenas $25 \%, 23 \%$ e $24 \%$, respectivamente. Relativamente ao estudo realizado em Portugal, Rodrigues et al. (2001) apresentam uma taxa de resposta de $21 \%$.

Segundo Pestana e Gageiro (2003), uma não resposta superior ou igual a $20 \%$ pode provocar um enviesamento dos resultados. Isso acontece se as empresas que não aceitaram participar tiverem características homogéneas e não dispersas, definindo assim uma categoria com características próprias. Essa ideia é confirmada por Young et al. (2005) ao referirem que a não resposta pode comprometer a generalização dos resultados para o universo que se pretende analisar. No entanto, estes autores consideram que esse problema está mais relacionado com a medida 
em que as empresas respondentes são diferentes das não respondentes, do que da própria taxa de resposta.

Neste trabalho, não se encontraram indícios de enviesamento provocado pelas empresas não respondentes, pelas seguintes razões:

- Em termos de cobertura geográfica do território português, estão representadas empresas de $79 \%$ dos distritos do universo, sendo que os três não cobertos tinham apenas uma empresa cada um. Assim o factor representatividade geográfica não é indicador de provocar enviesamento de resultados.

- Relativamente a uma distorção provocada pelo sector de actividade das empresas respondentes, confirmou-se existir uma grande dispersão de Classificação das Actividades Económicas $\square$ CAE das empresas entrevistadas. A mesma dispersão é encontrada nas não respondentes, o que também sugere não ser este um factor de enviesamento dos resultados.

- Por último, coloca-se a possibilidade de distorção provocada pelo factor dimensão das empresas, a qual, segundo Young et al. (2005), poder ser medida pelo número de trabalhadores. Foi realizado um estudo nas empresas não respondentes relativo ao número de trabalhadores. Verifica-se que o comportamento desta variável no grupo das não respondentes é idêntico ao do grupo das empresas entrevistadas. Para validar esta observação, utilizou-se o teste t student para a igualdade das médias, o qual apresenta um valor de 1,165, para 161 graus de liberdade, com um valor-p de 0,246, o que não permite rejeitar a hipótese nula de igualdade das médias. Diante dos resultados, podemos concluir que também não se recolheu evidência de que o factor dimensão possa provocar enviesamento dos resultados.

Esta análise permite-nos concluir que os resultados deste trabalho, embora não sendo válidos para todas as PMEs industriais portuguesas, podem caracterizar a realidade de um subconjunto dessas empresas, as classificadas como excelência de forma consistente em 2000 e 2001. Abernethy et al. (1999) concluem que, independente do método de investigação utilizado, a generalização a partir de um único trabalho é necessariamente limitada. Os autores consideram que a generalização em contabilidade interna tem de ser conseguida pela aplicação sucessiva em novas populações, locais e períodos temporais.

\section{Resultados}

Este ponto inicia-se com uma análise da forma como são repartidos os custos indirectos industriais, seguindo-se uma caracterização das bases de repartição utilizadas, e termina com a análise dos custos indirectos não industriais.

\section{a) - Métodos de repartição dos custos indirectos industriais}


Foi construída uma variável que reflecte não só o método de repartição dos custos indirectos industriais utilizado, mas também a sua não existência, denominada "custos indirectos industriais", a qual apresenta as quatro categorias de resposta evidenciadas no Quadro3:

- Em 24\% das empresas, não se repartem os custos indirectos industriais por produtos. No entanto, esse número esconde duas realidades distintas. Duas das empresas têm sistemas de contabilidade interna desenvolvidos, mas, por opção da gestão, utilizam apenas o custeio directo para valorizar os produtos; nas restantes 12 empresas, não se apura efectivamente o custo de cada produto.

- A maior frequência de resposta situa-se na utilização de uma única base de repartição (48\%). Nesse grupo, encontram-se bases como a hora-homem (Hh), hora-máquina $(\mathrm{Hm})$, quantidade produzida, percentagem dos custos directos, entre outras.

- Em 14\% das empresas, utilizam-se bases de repartição múltiplas, uma para cada grupo de custos indirectos. Nenhuma destas empresas utiliza mais de duas bases, sendo estas idênticas às referidas no grupo anterior.

- As restantes empresas (14\%) utilizam o método das secções homogéneas. É curioso observar que, mesmo nesse caso, se utilizam unidades de imputação idênticas às dos dois grupos anteriores, nomeadamente a hora-homem, horamáquina, quantidade produzida ou percentagem dos custos directos.

- Nenhuma das empresas utiliza ou está a implementar o custeio baseado nas actividades.

\section{Quadro3 - Variável "custos indirectos industriais"}

\begin{tabular}{||l|c|c|} 
Custos indirectos industriais & Frequências & Percentagens \\
\hline \hline Não repartem & 14 & $24 \%$ \\
Base única & 28 & $48 \%$ \\
Bases múltiplas & 8 & $14 \%$ \\
Método das seç̧ões & 8 & $14 \%$ \\
Custeio baseado nas actividades & 0 & $0 \%$ \\
\hline \hline Total & 58 & $100 \%$ \\
\hline
\end{tabular}

Fonte: Elaborado pela autora.

\section{b) - Bases de repartição}

Uma vez que as mesmas bases de repartição são utilizadas em base única, base múltipla ou mesmo como unidades de medida das secções, torna-se possível analisar quais as mais utilizadas, independente do sistema de repartição de custos indirectos. Pelo facto de muitas empresas utilizarem base múltipla, o número de casos analisados foi de 60, nas 44 empresas que repartem os custos indirectos 
industriais. O Quadro4 evidencia sete tipos de bases encontradas, todas elas sujeitas à influência do volume de produção.

- A base de repartição mais utilizada, com um peso de 35\%, é a horamáquina.

- Só em 27\% dos casos são encontradas bases de repartição relacionadas com a mão-de-obra directa. A hora-homem é mais utilizada (22\%) do que o valor da própria mão-de-obra directa $(5 \%)$.

- A seguir à mão-de-obra directa, as bases mais utilizadas são a quantidade produzida $(18 \%)$ e a proporção de custos directos $(15 \%)$.

- Só marginalmente foram encontradas outras bases de repartição como o valor das matérias consumidas, em dois casos, e o número de produtos da empresa, num caso. Esta última empresa tem apenas dois tipos de produtos e reparte os custos indirectos industriais em partes iguais para cada um deles.

Quadro4 - Tipos de bases de repartição

\begin{tabular}{||l|c|c||} 
Tipos de bases de repartição & Frequências & Percentagens \\
\hline \hline Hora-máquina $(\mathrm{Hm})$ & 21 & $35 \%$ \\
Hora-homem $(\mathrm{Hh})$ & 13 & $22 \%$ \\
Quantidade produzida & 11 & $18 \%$ \\
Percentagem dos custos directos & 9 & $15 \%$ \\
Valor da mão-de-obra directa & 3 & $5 \%$ \\
Valor das matérias consumidas & 2 & $3 \%$ \\
Outras bases & 1 & $2 \%$ \\
\hline \hline Total & 60 & $100 \%$ \\
\hline
\end{tabular}

Fonte: Elaborado pela autora.

A natureza real ou standard das bases foi analisada por Chun et al. (1996), por meio de inquéritos às práticas de contabilidade interna de 92 empresas industriais da Península da Malásia. Os resultados mostraram que as bases standard são muito utilizadas (36\%). Analisando as bases de repartição das empresas entrevistadas quanto à natureza standard ou real do seu valor, podemos concluir, pelo Quadro5, que a maioria (60\%) das bases utilizadas são standard.

\section{Quadro5 - Natureza das bases de repartição}

\begin{tabular}{|c|c|c|}
\hline Natureza das bases de repartição & Frequências & Percentagens \\
\hline Base real & 24 & $40 \%$ \\
\hline Base standard & 36 & $60 \%$ \\
\hline Total & 60 & $100 \%$ \\
\hline
\end{tabular}

Fonte: Elaborado pela autora.

A conjugação das duas informações anteriores permite analisar qual a na- 
tureza predominante (real ou standard) de cada uma das quatro bases de repartição mais utilizadas (Hm, Hh, produção, \% custos directos). Os resultados obtidos são apresentados no Quadro6 e traduzidos graficamente na Figura F1.

\section{Quadro6 - Tipo e natureza das bases de repartição}

\begin{tabular}{|l|l|c|c|c|}
\multicolumn{2}{|c||}{} & \multicolumn{2}{|c|}{ Natureza das bases repartição } & \multirow{2}{*}{ Total } \\
\cline { 3 - 4 } \multicolumn{2}{c|}{} & Base real & Base standard & \\
\hline \hline \multirow{2}{*}{$\begin{array}{c}\text { Tipos de bases de } \\
\text { repartição }\end{array}$} & Hora-máquina & 6 & 15 & 21 \\
\cline { 2 - 5 } & Hora-homem & 4 & 9 & 13 \\
\cline { 2 - 5 } & Quantidade produzida & 7 & 4 & 11 \\
\cline { 2 - 5 } & $\%$ custos directos & 3 & 6 & 9 \\
\hline \multicolumn{2}{|l|}{ Total } & 20 & 34 & 54 \\
\hline
\end{tabular}

Fonte: Elaborado pela autora.

Figura F1 - Tipo e natureza das bases de repartição

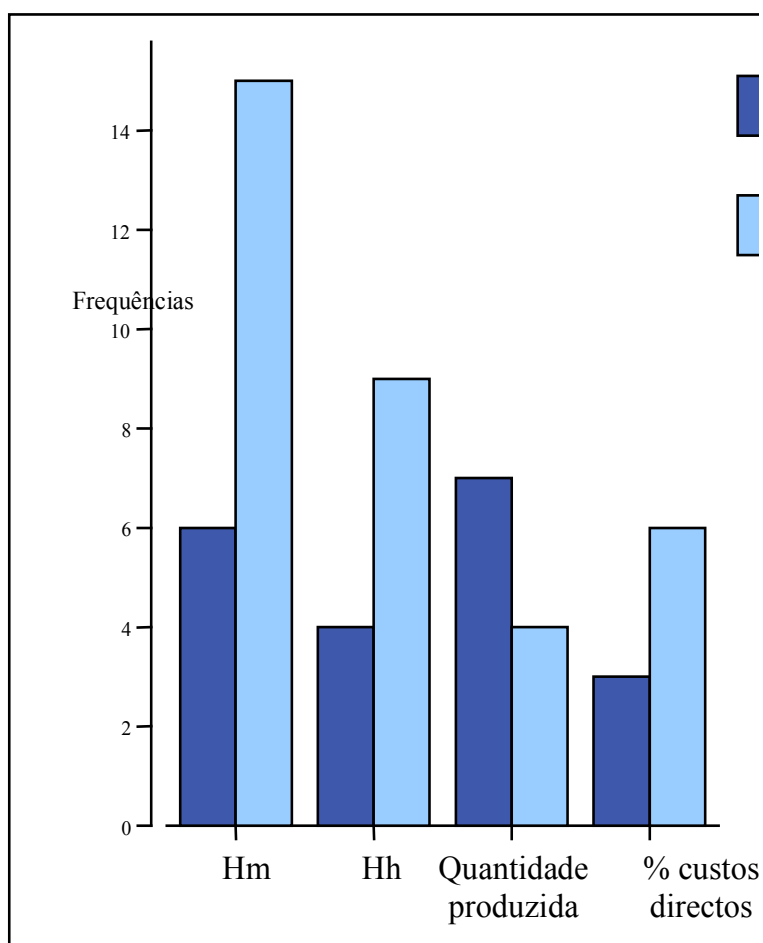

Fonte: Elaborada pela autora.

A sua análise permite concluir que:

- Nas empresas que utilizam bases de repartição reais, a mais utilizada é a quantidade produzida, seguindo-se a hora-máquina e a hora-homem.

- A única base de imputação que se utiliza mais em termos reais do que standard é a quantidade produzida. 
- Nas empresas que utilizam bases de imputação standard, a mais utilizada é a hora-máquina, seguida pela hora-homem, sendo a menos utilizada a quantidade produzida.

\section{c) - Repartição dos custos indirectos não industriais}

A repartição dos custos indirectos não industriais é ainda menos frequente nas empresas analisadas. O Quadro7 mostra que a maioria das empresas (57\%) não reparte esses custos por produtos. Revistas as entrevistas das 25 empresas que o fazem, verificou-se que todas utilizam uma única base para essa repartição. As bases utilizadas podem ser agrupados em três categorias:

- A mais utilizada, em nove das empresas, é o valor de vendas de cada produto.

- Em oito empresas, utiliza-se o custo das vendas de cada produto, como critério de repartição.

- As restantes oito empresas utilizam critérios diversificados, a maior parte relacionada com uma base de repartição teórica já utilizada para os custos indirectos industriais.

Quadro7 - Repartição dos custos indirectos não industriais

\begin{tabular}{||l|c|c||} 
Repartição custos indirectos não industriais & Frequências & Percentagens \\
\hline \hline Não repartem & 33 & $57 \%$ \\
Repartem & 25 & $43 \%$ \\
\hline \hline Total & 58 & $100 \%$ \\
\hline
\end{tabular}

Fonte: Elaborado pela autora.

\section{Conclusão}

Relativamente aos métodos de repartição dos custos indirectos industriais, a evidência recolhida mostrou que 14 das empresas analisadas (24\%) não os repartem por produtos. Das 44 que o fazem, a maioria (64\%) utiliza uma única base de repartição. Este é considerado como o método mais simples e menos rigoroso devido à dificuldade de encontrar uma relação de causa-efeito entre o comportamento de todos os custos indirectos e um único factor que seja adequado para os repartir. A taxa de utilização encontrada para a repartição de base única é muito superior à identificada em trabalhos realizados noutros países, revistos na terceira secção, que concluem que este método é utilizado, no máximo, por $35 \%$ das empresas. Ao contrário do reportado pelos estudos empíricos revistos na terceira secção, nenhuma das empresas analisadas utiliza ou está a implementar 
o custeio baseado nas actividades. A base de repartição mais utilizada é a horamáquina. Os estudos revistos na terceira secção permitem concluir que a base de repartição mais utilizada noutros países é a mão-de-obra directa, em valor ou em quantidade. Nas empresas analisadas, esta é a segunda base mais utilizada. Todas as bases de repartição encontradas são influenciadas pela quantidade produzida de cada produto. São os que têm maior volume de produção que mais consomem destas bases, logo são valorizados com uma parcela maior de custos indirectos. Caso existam custos não relacionados com o volume de produção, a utilização destas bases de repartição pode não reflectir o verdadeiro consumo de recursos organizacionais requeridos por cada produto, gerando a sobrevalorização dos que são fabricados em grandes quantidades e a subvalorização dos que são produzidos em pequenos lotes. Se o custo dos produtos daqui resultante for utilizado para efeitos de tomada de decisão, nomeadamente para determinação do preço de venda ou análise de rendibilidade de cada produto, pode ter implicações estratégicas importantes na empresa na medida em que os gestores podem conduzir erradamente os recursos para os produtos com menor volume de produção. $\mathrm{O}$ facto de a maioria das empresas não repartir por produtos os custos indirectos não industriais não nos permite retirar conclusões relevantes quanto à natureza das bases de repartição utilizadas para esses custos.

\section{REFERÊNCIAS:}

ABERNETHY, Margaret A., e CHUA, Wai Fong, e LUCKETT, Peter F.; SELTO, Frank H. Research in managerial accounting: Learning from others' experiences. Accounting and Finance, $n^{\circ}$ 39, p. 1-27, 1999.

BELL, Judith. Como realizar um projecto de investigação. 2. ed. Lisboa: Gradiva, 2002.

BHIMANI, Alnoor. European management accounting research: traditions in the making. The European Accounting Review, Volume 11, no 1, p. 99-117, 2002.

BLAKE, John; WRAITH, Philip; AMAT, Oriol. Developing a new national management accounting framework - the Spanish case. European Business Review, $\mathrm{n}^{\mathrm{o}} 12-3, \mathrm{p} .122-128,2000$.

BURNS, John; SCAPENS, Robert W. Conceptualizing management accounting change: an institutional framework. Management Accounting Research, $n^{\mathbf{o}} 11$, p. 3-25, 2000. 
CHAMBERS, R. J. Positive Accounting Theory and the PA Cult. ABACUS, Volume 29, nº1, p.1-26, 1993.

CHAUVEY, Jean-Noël. La méthode ABC à l'épreuve des faits. Revue Française de Comptabilité, no 294, p. 53-62, 1997.

CHUA, Wai Fong. Radical developments in accounting thought. The Accounting Review, Vol. LXI, nº 4, p. 601-632, 1986.

CHUN, Loo Sin; KASSIM, Nor Aziah H. A.; MINAI, Badriyah. Are management accounting systems in Malaysia outmoded? Singapore Management Review, volume $18, \mathrm{n}^{\mathrm{o}} 1$, p. 55-67, 1996.

CLARKE, Peter J.; O’ DEA, Tony. Management accounting systems: some field evidence from sixteen multi-national companies in Ireland. Irish Accounting Review, Spring, p.199-216, 1994.

CLARKE, Peter J. Management accounting practices in large Irish manufacturing firms. IBAR- Irish Business and Administrative Research, $\mathrm{n}^{\mathbf{0}}$ 18, p. 136-152, 1997.

CLARKE, Peter J., Hill; NANCY, Thorley; STEVENS, Kevin. Activity-Based Costing in Ireland: Barriers to, and opportunities for, change. Critical Perspectives on Accounting, $\mathrm{n}^{\text {o }}$ 10, p. 443-468, 1999.

COOPER, Robin. The Two-Stage Procedure in Cost Accounting: Part One. Journal of Cost Management, Summer, p. 43-51, 1987a.

COOPER, Robin. Does Your Company Need a New Cost System? Journal of Cost Management, Spring, p. 45-49, $1987 \mathrm{~b}$.

COOPER, Robin. The Rise of Activity-Based Costing - Part One: What Is an Activity-Based Cost System? Journal of Cost Management, Summer, p. 45$54,1988$.

COTTON, Bill; JACKMAN, Susan. Activity based costing: not a "easy ABC". Chartered Accountants Journal of New Zealand, no 81- 4, p. 35-37, 2002.

COVALESKI, Mark A.; DIRSMITH, Mark W. An Institutional Perspective on the Rise, Social Transformation, and Fall of a University Bubget Category. Administrative Science Quarterly, no 33, p. 562-587, 1988.

DEARMAN, David T.; SHIELDS, Michael D. Cost knowledge and cost-based judgment performance. Journal of Management Accounting Research, $\mathrm{n}^{\mathbf{0}} 13$, 
p. 1-18, 2001.

DRURY, Colin; TAYLES, Mike. Product costing in UK manufacturing organizations. The European Accounting Review, no 3:3, p. 443-469, 1994.

FERREIRA, Rogério Fernandes. Opinar sobre contabilidade, Jornal de Contabilidade, Ano XXXI, nº 361, p. 116-117, 2007.

FRANCO, Victor Seabra et al. Contabilidade de Gestão - Volume I. O apuramento do custo dos produtos e a informação de apoio à decisão. Lisboa: Publisher Team, 2005.

GHOSH, B. C.; CHAN, Yoke-Kai. Management accounting in Singapore well in place? Managerial Auditing Journal, volume 12, nº 1, p.16-18, 1997.

GOSSELIN, Maurice. The effect of strategy and organizational structure on the adoption and implementation of activity-based costing. Accounting, Organizations and Society, $\mathrm{n}^{\mathrm{o}}$ 22, p. 105-122, 1997.

HALDMA, Toomas; LÄÄTS, Kertu. Contingencies influencing the management accounting practices of Estonian manufacturing companies. Management Accounting Research, $\mathrm{n}^{\mathrm{o}}$ 13, p. 379-400, 2002.

HOPPER, Trevor; ARMSTRONG, Peter. Cost accounting, controlling labour and the rise of conglomerates. Accounting, Organizations and Society, $\mathrm{n}^{\mathrm{o}} 16$, p. 405-438, 1991.

HOPPER, Trevor; KOGA, Tsutomu; GOTO, Jitsuo. Cost accounting in small and medium sized Japanese companies: an exploratory study. Accounting and Business Research, volume 30, nº 1, p. 73-86, 1999.

HORNGREN, Charles T.; FOSTER, George; DATAR, Srikant M. Cost accounting. A managerial emphasis. 9. ed. London: Prentice Hall International, 1997.

HORNGREN, Charles T. Management accounting: some comments. Journal of Management Accounting Research, $n^{\circ}$ 16, p. 207-211, 2004.

HUMPHREY, Christopher; SCAPENS, Robert W. Methodological themes. Theories and case studies of organizational accounting practices: limitation or liberation? Accounting Auditing \& Accountability Journal, Volume 9, no 4, p. 86-106, 1996.

IAPMEI. Estrutura Empresarial Nacional. Lisboa: Gabinete de Estudos do Instituto de Apoio às Pequenas e Médias Empresas e ao Investimento, 2002. 
INNES, John; MITCHELL, Falconer. A survey of activity-based costing in the U.K.'s largest companies. Management Accounting Research, $n^{\circ} 6$, p. 137-153, 1995.

INNES, John; MITCHELL, Falconer; SINCLAIR, Donald. Activity-based costing in the U.K.'s largest companies: a comparison of 1994 and 1999 survey results. Management Accounting Research, $n^{\text {o }}$ 11, p. 349-362, 2000.

JOHNSON, H. Thomas. Activity-Based Information: A Blueprint for World-class Management accounting. Management Accounting, June, p. 23-30, 1988.

JOHNSON, H. Thomas; KAPLAN, Robert S. Relevance Lost: The Rise and Fall of Management Accounting, 2. ed. Boston: Harvard Business School Press, 1991.

JONES, T. Colwyn; DUGDALE, David. The ABC bandwagon and the juggernaut of modernity. Accounting, Organizations and Society, $n^{\circ} 27$, p. 121-163, 2002.

JOSHI, P. L. The international diffusion of new management accounting practices: The case of India. Journal of International Accounting Auditing \& Taxation, volume 10, $\mathrm{n}^{\mathrm{o}} 1$, p. 85-109, 2001.

KAPLAN, Robert S. Innovation Action Research: Creating New Management Theory and Practice. Journal of Management Accounting Research, $n^{\circ} 10, p$. 90-117, 1998.

KENNEDY, Tom; AFFLECK-GRAVES, John. The Impact of activity-Based Costing Techniques on Firm Performance. Journal of Management Accounting Research, $\mathrm{n}^{\circ}$ 13, p. 19-45, 2001.

LAUGHLIN, Richard C. Accounting systems in organisational contexts: a case for critical theory. Accounting, Organizations and Society, $n^{\circ} 12$, p. 479-502, 1987.

LEBAS, Michel. Managerial accounting in France. Overview of past tradition and current practice. The European Accounting Review, nº 3:3, p. 471-487, 1994.

LUKKA, Kari; GRANLUND, Markus. Cost accounting in Finland: current practice and trends of development. The European Accounting Review, Volume 5, $\mathrm{n}^{\mathrm{o}} 1$, p. 1-28, 1996.

. The fragmented communication structure within the accounting academia: the case of activity-based costing research genres. Accounting, Organizations 
and Society, $\mathrm{n}^{\mathrm{0}} 27$, p. 165-190, 2002.

MAJOR, Maria, e HOOPER, Trevor. Managers divided: implementing ABC in a Portuguese Telecommunications Company. Management Accounting Research, $n^{\circ} 16$, p. 205-229, 2005.

MEVELLEC, Pierre. Les difficultés de conception et de mise en oeuvre de systèmes ABC. Revue Française de Comptabilité, nº 338, p.1-4, 2001.

MILLER, Peter; O' LEARY, Ted. Accounting and the construction of the governable person. Accounting, Organizations and Society, n ${ }^{\circ}$ 12, p. 235-265, 1987.

PEREIRA, Carlos Caiano; FRANCO, Victor Seabra. Contabilidade Analítica, Lisboa: Edição particular, 1991.

PESTANA, Maria Helena; GAGEIRO, João Nunes. Análise de dados para ciências sociais. A complementaridade do SPSS, 3. ed. Lisboa: Edições Sílabo, 2003.

RODRIGUES, Ana Isabel et al. Estado da contabilidade de gestão nas PME's Portuguesas. Resultados de um estudo empírico, Jornal do Técnico de Contas e da Empresa, Abril, p. 471-475, 2001.

SCAPENS, Robert W. Researching Management Accounting Practice: The Role of Case Study Methods. British Accounting Review, n 22, p. 259-281, 1990.

SWENSON, Dan. The Beneficts of Activity-Based Cost Management to the Manufacturing Industry. Journal of Management Accounting Research, $\mathrm{n}^{\mathbf{0}} 7$, p. 167-180, 1995.

YOUNG, S. Mark; WIM, A. Van der Stede; CHEN, Clara Xiaoling. Assessing de quality of evidence in empirical management accounting research: the case of survey studies. Accounting, Organizations and Society, n 30, p. 655-684, 2005.

WATTS, Ross L.; ZIMMERMAN, Jerold L. Positive Accounting Theory: A Ten Year Perspective. The Accounting Review, Volume 65, nº 1, p. 131-156, 1990.

ZIMMERMAN, Jerold L. Conjectures Regarding Empirical Managerial Accounting Research. Journal of Accounting and Economics, $n^{\circ} 32$, p. 411-427, 2001. 
